\title{
Integrated modelling of ICRH in a quasi-axisymmetric stellarator
}

\author{
M. Jucker ${ }^{1}$, W.A. Cooper and J.P. Graves \\ Ecole Polytechnique Fédérale de Lausanne (EPFL), Centre de Recherches en Physique des \\ Plasmas, Association EURATOM-Confédération Suisse, CH-1015 Lausanne, Switzerland \\ E-mail: mjucker@princeton.edu
}

Received 13 July 2011, accepted for publication 23 November 2011

Published 23 December 2011

Online at stacks.iop.org/NF/52/013015

\begin{abstract}
We apply the code package SCENIC to a two field-period quasi-axisymmetric stellarator. Ion cyclotron resonance heating (ICRH) is applied both on the high- and low-field side to a $1 \%{ }^{3} \mathrm{He}$ minority in a deuterium plasma. It is shown that due to toroidal variations, the results are considerably different from similar tokamak studies. In particular, toroidal variations in power deposition and pressure are created and accentuated during radio frequency heating, such that modifications to the magnetic equilibrium depend on toroidal angle. We demonstrate that due to enhanced particle loss, low-field side heating is significantly less efficient than high-field side heating, and that toroidally trapped particles impose upper power limits for efficient radio frequency injection.
\end{abstract}

(Some figures may appear in colour only in the online journal)

\section{Introduction}

Quasi-axisymmetric stellarators (QASs) represent feasible combinations of tokamaks and stellarators, combining the best features of each configuration. On the one hand, the threedimensional geometry allows for a plasma without toroidal current, thus improving considerably MHD stability and allowing for disruption free steady state. On the other hand, and in contrast to conventional stellarators, the magnetic field strength depends only on two variables, a radial and an angular variable, such that the particle orbits stay confined tokamaklike around constant pressure surfaces [1]. Furthermore, controlling a desired asymmetry in toroidal direction of a stellarator can be more efficient than trying to suppress magnetic field ripple and other inhomogeneities in a tokamak, and it adds one more dimension for plasma stability control [2].

Until now, self-consistent solutions of the wave fields in the ion cyclotron resonance heating (ICRH) spectrum and fast particle distribution solvers have been limited mostly to two-dimensional tokamak plasmas which fail to include the modifications to the equilibrium state [3-6]. In threedimensional magnetically confined plasmas, the TASK/WM field solver has been coupled with the GNET drift kinetic equation solver, but the equilibrium modifications have been excluded from the iterative loop [7].

We apply the unique code package SCENIC [8] to a two-field period QAS, in order to study ICRH in a threedimensional equilibrium. SCENIC generates self-consistent

${ }^{1}$ Present address: Princeton University, AOS Program, Princeton, NJ 08544, USA. three-dimensional solutions, which include varying MHD equilibrium, radio frequency (RF) wave field and resonant particle distribution functions, through the guise of an iterative scheme. To our knowledge, this is the first time such comprehensive ICRH simulations are performed in a threedimensional geometry. In the next section, we will introduce the numerical setup, before reporting our simulation results in section 3 , and finish with concluding remarks.

\section{Numerical model and geometry}

\subsection{SCENIC}

The fully three-dimensional code package SCENIC has been described extensively in [8], and we will only give a general overview for the clarity of this work. SCENIC is an iterative scheme and comprises three codes plus additional modules required for the iterations. The MHD equilibrium code VMEC $[9,10]$ computes a new anisotropic fixed boundary equilibrium after each iteration. Then, the full-wave code LEMan $[11,12]$ reads the newly created equilibrium and updates the RF wave field. Finally, VENUS [13,14] advances the distribution function based on the updated equilibrium and wave field, and re-creates new inputs for the next iteration of VMEC and LEMan. Wave-particle interactions are modelled in a Monte Carlo approach, and the iterative scheme can be run until convergence is achieved, and total input RF power equals total power going to the background plasma plus power loss due to energetic particles crossing the plasma boundary. 


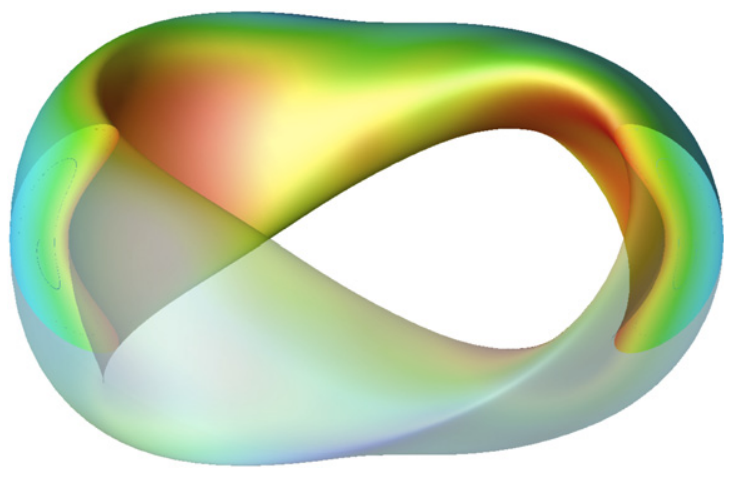

Figure 1. Initial magnetic equilibrium for the two-period QAS. The magnetic field varies from $B_{\min }=2.0 \mathrm{~T}$ (blue) to $B_{\max }=3.2 \mathrm{~T}$ (red), with the on axis magnitude of $B_{0}=2.57 \mathrm{~T}$. The poloidal cuts are at $\phi=\{0, p i\}$.

\subsection{Quasi-axisymmetric stellarator}

We investigate a two-field period QAS of size comparable to the Joint European Torus (JET). This is a rescaled version of CHS-qa, a design proposed in Japan [15], and its magnetic equilibrium studied numerically in [16]. This particular choice of geometry is motivated by recent work $[8,17]$, which applies the SCENIC package to a JET-like two-dimensional torus. It is interesting to consider a three-dimensional geometry with as many similar parameters as possible, as it allows for a more direct comparison between tokamak and stellarator simulations. Figure 1 illustrates the magnetic field strength, which varies in the range $2.0 \mathrm{~T} \leqslant B \leqslant 3.2 \mathrm{~T}$. The toroidal angle is defined to have its origin to the left of the figure, and the plasma volume is semi-transparent between $\phi=0$ and $\pi=2 \pi / L$, with $L=2$ the number of field periods. We chose to match major $\left(R_{0}=2.91 \mathrm{~m}\right)$ and minor $(a=0.89 \mathrm{~m})$ radii rather than plasma volume $\left(37.3 \mathrm{~m}^{3}\right)$, as well as magnetic field strength on axis $\left(B_{0}=2.57 \mathrm{~T}\right)$. The safety factor varies only very little around $q \approx 3$, and the volume averaged thermal beta is $\langle\beta\rangle=0.5 \%$. Whereas we could have pushed for higher thermal beta, there are two good reasons for our choice: first, as mentioned above, our approach is to take previous results one step further by adding a third dimension to the studies performed in [8]. Second, we are mainly interested in fast (resonant) particle effects, and therefore do not necessarily need high thermal pressure. As for the geometry, note that the plasma volume is about half of JET's plasma volume even with comparable major and minor radii, which is due to the toroidal plasma shaping of the QAS. The electron density profile was chosen $n=n_{0}\left(1-s^{4}\right)$ with $n_{0}=3 \times 10^{19} \mathrm{~m}^{-3}$, and the temperature profile $T=T_{0}(1-s)$ with $T_{0}=3 \mathrm{keV}$. Here, $s$ is the Boozer radial coordinate and is proportional to toroidal flux. The plasma is composed of $1 \%$ helium- 3 in a deuterium background. The choice of helium- 3 is motivated by the fact that it prevents the appearance of a deuterium second harmonic resonance in the plasma, and for the sake of comparison with tokamak simulations in [17]. In the latter studies, helium-3 minority was chosen as it is relevant for reactor-like scenarios (such as ITER).

Figure 2 represents an artist's view of the QAS, including stylized magnetic field coils (black), an example particle orbit (in red), and the magnetic field isosurface corresponding to

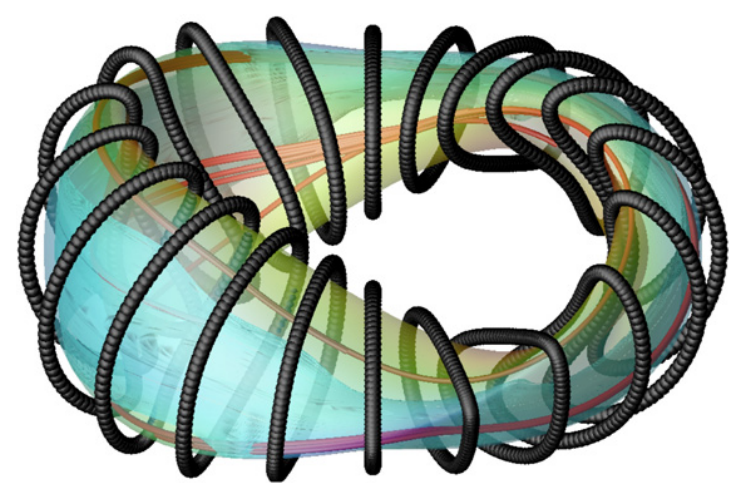

Figure 2. Artist's view of the studied QAS. It features an example particle orbit, the cold resonant layer (low-field side), and stylized magnetic field coils. We stress that computations are performed with fixed boundary, and the field coils added for easier recognition of the plasma shape.

the cold low-field side (LFS) resonance $(B=2.35 \mathrm{~T})$, which we will define in more detail later. Note that the field coils have been added for emphasizing the plasma shape, but computations are based on a fixed boundary. Enclosed in this figure is one example of the effects of the three-dimensional geometry of the device studied: the orbit contains a time frame where the particle is trapped toroidally on the high-field side (HFS) of the poloidal plane. Figure 3 zooms in on the relevant section of figure 2. The particle is indeed trapped between maxima of the magnetic field when moving along the toroidal angle. Figure 3(a) focuses on the part of the orbit in which the toroidal trapping appears. The coloured shading corresponds to the magnetic field strength along the plasma boundary. One can see that the particle is trapped between the two regions of maximum field strength shaded in red in the figure. In order to prove that the particle is indeed trapped toroidally and not poloidally, we plot the poloidal and toroidal angles as a function of time in figure $3(b)$. The particle emerges from the inboard midplane $(\theta=\pi)$, and is then trapped with $\theta>\pi / 2$ (i.e. on the HFS), before it de-traps and moves on towards the LFS $(\theta<\pi / 2)$. Trapping and de-trapping of this particular orbit in time is due to the combined effects of Coulomb collisions and RF interactions, changing the pitch angle and energy of the particle.

\subsection{ICRH setup}

We apply external heating at two different frequencies. SCENIC is restricted to fundamental minority heating, such that the frequency is uniquely defined by the resonant species $\left({ }^{3} \mathrm{He}\right)$ and the resonant magnetic field strength $B_{\mathrm{c}}$ by $\omega=$ $Z e B_{\mathrm{c}} / A m_{\mathrm{p}}$, where $Z$ and $A$ are the atomic charge and mass of the minority species, and $m_{\mathrm{p}}$ the proton mass. We chose $B_{\mathrm{c}}=2.8(2.35) \mathrm{T}$ for HFS (LFS) heating, such that the cold resonant layer is about half way between the magnetic axis and the edge along the midplane, yielding frequencies of $f=28.5$ (23.9) $\mathrm{MHz}$, respectively.

For simplicity, the RF antenna is chosen to be localized in poloidal angle only, and placed on the LFS wall all around the plasma in the toroidal direction. The antenna is exciting one simple toroidal mode number $n=17$ representing the peak of a realistic mode spectrum parallel to the toroidal magnetic 


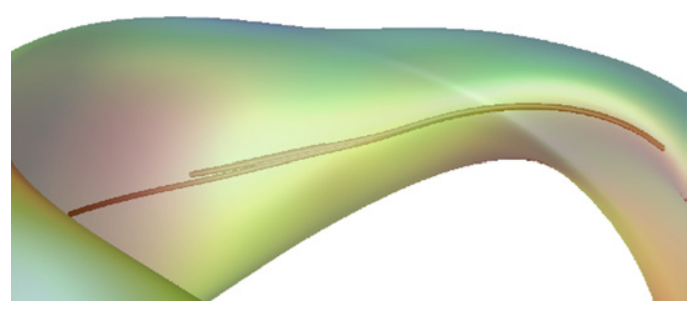

(a) Part of the orbit of figure 2 where the particle is trapped on the HFS between field maxima along the toroidal angle (red shaded).

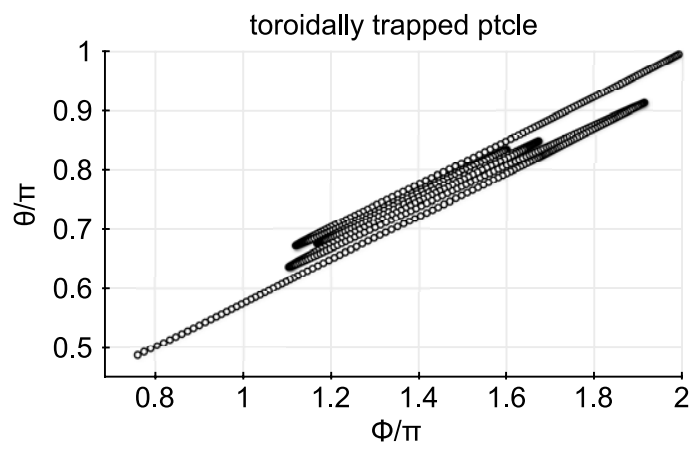

(b) View in $(\theta, \phi)$ space. The particle stays for some time trapped with $\theta \in$ $(\pi / 2, \pi)$, i.e. on the high-field side of the plasma.

Figure 3. Focus on the part of an orbit where the particle is toroidally trapped on the HFS of the poloidal plane. The particle motion depicted in $(b)$ starts at $(\theta=\pi, \phi=2 \pi)$ (upper right) and evolves to $(\theta<\pi / 2, \phi<4 \pi / 5)$ (lower left) in time.

field. Note that the wave code LEMan is capable of dealing with interacting toroidal modes in a full three-dimensional spectrum, but we decided to concentrate on the novelty of being able to compute self-consistent distribution functions and equilibria in fully shaped three-dimensional geometries with SCENIC, keeping all other model extensions as simple as possible. For the same reason, we start each simulation with a completely thermalized ${ }^{3} \mathrm{He}$ minority, having the same density and temperature profiles as the background deuterium. The only difference under the initial conditions is that the deuterium density is $n_{0}^{\mathrm{D}}=0.98 n_{0}$, whereas helium-3 density is $n_{0}^{{ }^{3} \mathrm{He}}=$ $0.01 n_{0}$, giving the $1 \%$ minority concentration. Of course, all helium-3 profiles can evolve freely once the simulation started. Note also that the parallel and perpendicular wave numbers are computed consistently with the wave field within LEMan, and no approximation is needed, such that e.g. wave number upshift can be included and no explicit dispersion relation has to be invoked.

Earlier studies in JET-like two-dimensional tokamaks revealed that the highly energetic tails of the distribution function become most important when heating is applied on the LFS, and diminish steadily when moving towards the HFS [17]. In these studies, on axis heating results were somewhere in-between the HFS and LFS cases, and therefore the main focus lies on the two off-axis positions. Although in this work we do not provide direct comparisons between the tokamak and the stellarator results, we will limit our stellarator studies to scenarios as similar as possible to the tokamak simulations, and not consider central heating. LFS heating can be expected to have much more localized effects than HFS, and as a consequence can drive MHD instabilities through strongly enhanced pressure gradients [18]. In contrast, HFS heating can heat the plasma evenly along the resonant layer, and resonant particles lose more of their energy to the background plasma, increasing the background plasma heating efficiency. It is therefore interesting to investigate the RF heating effects on distribution function, wave field and plasma equilibrium for both scenarios. We will show here that in the chosen scenarios, HFS and LFS heating are substantially dissimilar, and differ from the tokamak studies mostly in the LFS scenario.
In any stellarator, most physical quantities depend on the toroidal angle as well as radial position and poloidal angle. We focus on two different toroidal angles, one at $\phi=0$, the other at $\phi=2 \pi / 2 L$, where $L=2$ is the number of toroidal periods. These two angles mark the two extremes in plasma shaping along the toroidal direction, and seem therefore natural choices. At these toroidal angles, we will show twodimensional plots in $(R, Z)$ coordinates wherever possible, such that all geometric dependencies can be included.

\section{ICRH simulations}

In all simulations, $4 \times 10^{6}$ markers were followed in VENUS. Each simulation is stopped if at the end of a given iteration the power balance shows that fast particle energy content is stationary. This is determined by the balance between input RF power (heating), power going from the fast ions to the background plasma through collisions (cooling), and power loss due to fast particles crossing the plasma boundary. The simulations for $6 \mathrm{MW}$ (3 MW) HFS converged after 16 (12) iterations of $25 \mathrm{~ms}$ each, resulting in a total simulation time of 2.7 (2.0) electron slowing down times. The LFS results were obtained with a total simulation time of three slowing down times.

\subsection{High-field side}

The applied RF power is varied between moderate $3 \mathrm{MW}$ (which would be equivalent to $6 \mathrm{MW}$ in JET due to its larger plasma volume) and rather high 6 MW (equivalent to $12 \mathrm{MW}$ in a JET sized plasma volume), keeping all other parameters constant. The main difference is that the $6 \mathrm{MW}$ runs give a more pronounced picture of the mechanisms at play, but qualitatively they are very similar. We will therefore concentrate on the higher power simulations, and only point out differences where they are non-trivial.

3.1.1. Effective temperature. At $3 \mathrm{MW}$, in steady state half of the input power is passed on to the background electrons and ions, and half is lost through the plasma boundary. About $12 \%$ of the helium-3 minority is in the non-Maxwellian tail 


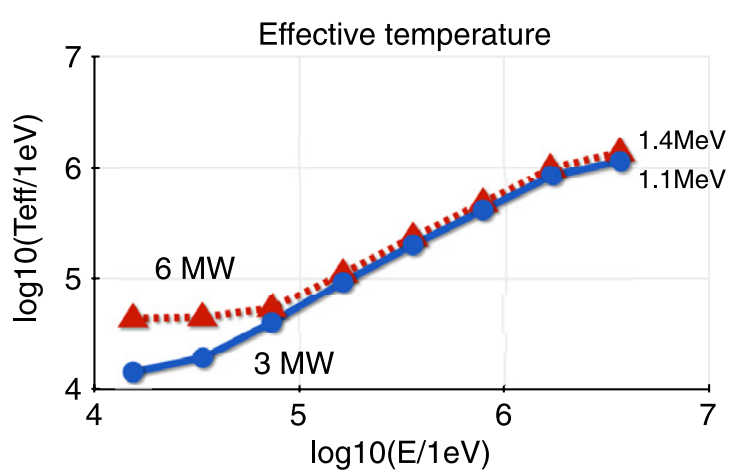

Figure 4. Effective temperatures defined in (1) for the 3 and $6 \mathrm{MW}$ cases. Whereas they are very similar at energies above $100 \mathrm{keV}$, they diverge in the mid-range $10-100 \mathrm{keV}$. The main difference between these two regions in energy space is the slowing down, which is entirely on electrons for high energy, and on ions at lower energies. Note that a Maxwellian would be represented by a horizontal line in this plot.

of the distribution function, whereas that fraction increases to $16 \%$ for $6 \mathrm{MW}$ heating. For the latter, only about $40 \%$ of the input power is going to the plasma, and $60 \%$ lost. In order to investigate the differences in the distribution function, we define the effective temperature as

$$
T_{\text {eff }}(E)=-\left[\frac{\mathrm{d} \ln f(E)}{\mathrm{d} E}\right]^{-1},
$$

and treat it as a measure of how energetic the tail of the distribution function is. Note that this is a purely diagnostic quantity, as it does not consider differences in perpendicular and parallel directions, and we will apply the derivative not to the spatially local distribution function, but the real space and pitch angle integrated distribution function $f(E)$. Figure 4 plots the ${ }^{3} \mathrm{He}$ effective temperatures for the 3 and $6 \mathrm{MW}$ cases in the non-Maxwellian region above $10 \mathrm{keV}$. We would like to point out two features. First, the effective temperatures for energies higher than $100 \mathrm{keV}$, where slowing down is on electrons only, are very similar for both power cases. This means that even though there is a difference in input power, the very high energy tails of the distribution function may be smaller in terms of density, but they assume the same form, with the same tail temperatures. This would suggest that the very high energy tail temperatures are not influenced by the heating power, but rather the electron density profile (slowing down) and particle losses. Second, in the intermediate energy range between 10 and $100 \mathrm{keV}$, effective temperature differences are very obvious. This is the region where collisions on background ions are nonnegligible, and increased RF power allows for higher effective temperatures.

3.1.2. Current density. An important quantity for both diagnostics and equilibrium computations is the first moment of the distribution function, the toroidal current density, and is plotted in figure 5(a) (without any drag current corrections). In the QAS, ohmic current is zero. In addition, the model does not include any modifications of the bulk ion and electron profiles, and therefore bootstrap current modifications arising from thermal profile changes are not considered here. As under the initial conditions, bootstrap current was set to zero as well, this plot represents at the same time the total toroidal current density in our simulations. Note that we plot here the total current density, whereas the drag current corrected density is fed to the equilibrium calculation in the iterative scheme [8]. As expected, the current is considerably larger in the $6 \mathrm{MW}$ scenario, yielding a total toroidal current of about $4.5 \mathrm{kA}$, whereas the integrated toroidal current in the $3 \mathrm{MW}$ scenario amounts to $1 \mathrm{kA}$. Note how the current densities are peaked just inside the cold resonant layer $\sqrt{s} \approx 0.5$, and that the higher power case contains a secondary maximum close to the magnetic axis. We attribute this to non-standard orbits of highly energetic particles close to the magnetic axis [19]. The current induced change in the safety factor (figure $5(b)$ ) is of the order of $0.1-0.5 \%$, and we conclude that the RF driven current in these scenarios has a rather low impact on the magnetic equilibrium.

3.1.3. Distribution function and power deposition. We will now investigate if there are any differences in the toroidal direction of the QAS. Figure 6 plots and compares the $f\left(\rho_{\mathrm{c}}, \theta=0, \phi=\{0, \pi / 2\}, v_{\|}, v_{\perp}\right)$ contours of the distribution function for the more extreme $6 \mathrm{MW}$ case. $\rho \equiv \sqrt{s}$ corresponds to $r / a$ in a tokamak, with $r$ local minor and $a$ maximum minor radii, and $\rho_{\mathrm{c}}$ is the radial position of the cold resonant layer on the midplane. The contour plots do not show important dependencies on $\phi$ in the distribution function if one follows otherwise constant Boozer coordinates. The resolution in phase space is, however, rather low, due to the fact that the exact value of the distribution function in phase space is not needed in the numerical scheme, and is only computed for diagnostics reasons [8]. However, these plots confirm the findings of figure $5(a)$, in that there is an asymmetry between positive and negative parallel velocities, resulting in the observed positive (i.e. parallel to the toroidal magnetic field) toroidal currents. Positive toroidal current generation is expected, as the wave spectrum is represented by one cocurrent toroidal mode number.

Changes in the distribution function discussed above are induced by wave-particle interactions between the helium3 minority and the injected electromagnetic wave. The spatial distribution of these interactions can be visualized most conveniently by the help of the three-dimensional power deposition within the plasma. Figure 7 shows the power deposition in the Monte Carlo RF operators of SCENIC (VENUS) in poloidal cuts at six different toroidal positions along one half field period. The top panel shows the initial state, where the deposition is rather well distributed along toroidal angle, with a somewhat distinct maximum near $\phi=0$ (due to the setup of the numerical diagnostics, it is not possible to show these plots at exactly $\phi=0$ or $\pi / 2$ ). The situation is more extreme at the end of the simulation on the bottom panel, where the absolute maximum near $\phi=0$ is much larger, and dominates the power deposition completely. Even if the antenna is perfectly symmetric in the toroidal direction, the power deposition assumes a dependence on $\phi$, peaking around the extremities of each field period, and this dependence gets stronger as the plasma is heated with RF waves. On the one hand, heating is strongest where the plasma is thinnest in terms of major radius, and the RF wave has to propagate 


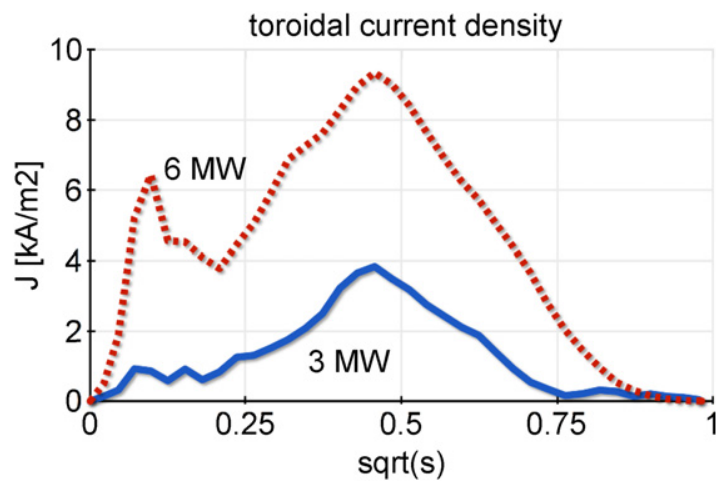

(a) Toroidal current density. For the higher power, the total toroidal current is about $4.5 \mathrm{kA}$, whereas the lower $\mathrm{RF}$ power generates a toroidal current of $1 \mathrm{kA}$.

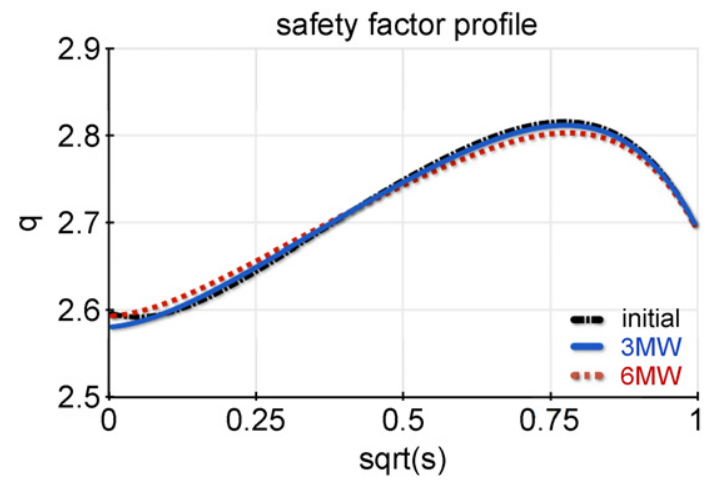

(b) Safety factor profile. In black and dashdotted the initial profile. Modifications of the safety factor amount to about $0.5 \%(0.1 \%)$ for $6 \mathrm{MW}(3 \mathrm{MW})$ power.

Figure 5. Toroidal current density and safety factor profiles for the 3 and $6 \mathrm{MW}$ scenarios.

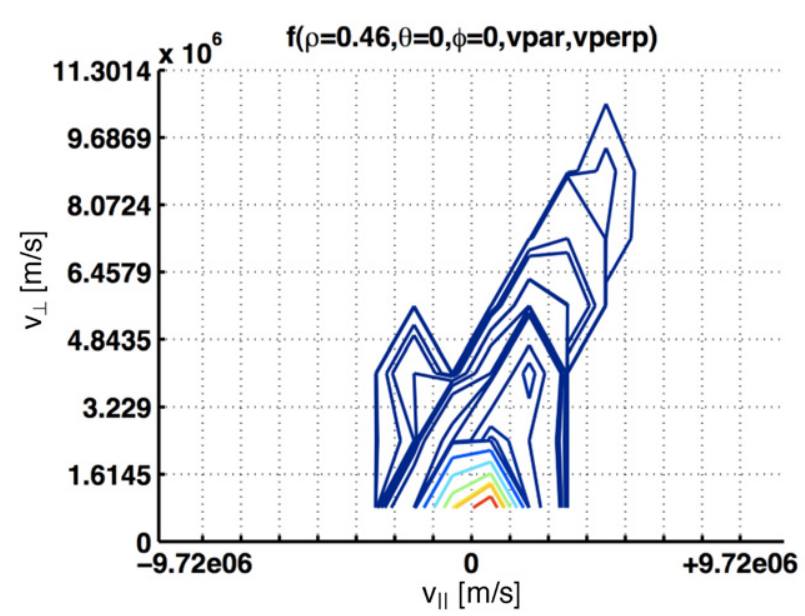

(a) $f\left(v_{\|}, v_{\perp}\right)$ at $\phi=0$.

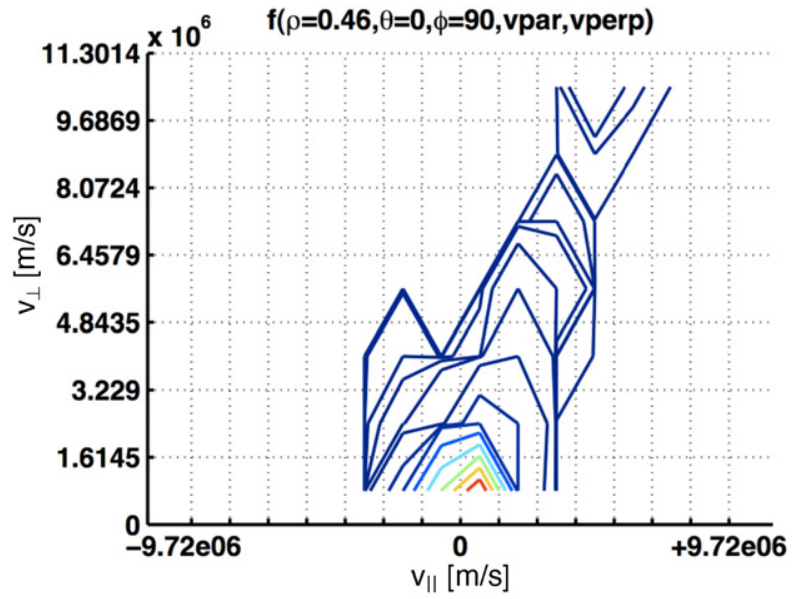

(b) $f\left(v_{\|}, v_{\perp}\right)$ at $\phi=\pi / 2$.

Figure 6. Contour plots for $6 \mathrm{MW}$ HFS heating. The energetic particles make about $16 \%$ of the ${ }^{3} \mathrm{He}$ minority. Both plots show very similar asymmetries for high energies and $v_{\perp}>v_{\|}>0$, resulting in positive currents.

smaller distances in the plasma before being absorbed at the resonant layer. This is true for initial as well as the final state in the simulation. A second geometric effect that could favour absorption is the large elongation at the $\phi=(0, \pi)$ planes. As the distribution of the magnetic field strength is tokamak-like for the configuration examined, the nearly vertical resonant layer is longest on these respective cross sections, allowing for more power deposition. On the other hand, and more importantly, when moving along the toroidal direction, the magnetic field is strongest around $\phi=\{0, \pi\}$ (see figures 1 and 15). As a result, toroidally trapped particles have their turning points around these aforementioned toroidal angles. In addition, the turning points are the locations where wave-particle interaction is strongest as particles stay in resonance for a longer time. At the turning points, the perpendicular velocity is much higher than the parallel velocity, resulting in locally enhanced pressure anisotropy. We will now examine if this non-uniform absorption translates into toroidally dependent pressure and magnetic field strength.
3.1.4. Pressure. Parallel and perpendicular pressures are the second moments of the distribution function. As we will see, it is important to treat them separately, as pressure anisotropy forms in RF heating scenarios and can have important effects on equilibrium and stability [20,21]. Figure 8 plots the hot particle perpendicular and parallel pressure at three different toroidal locations, going from the above seen maximum power deposition at $\phi=0$ to the minimum at $\phi=\pi / 2$. Whereas the hot parallel pressure does not show any significant variation, the hot perpendicular pressure maximum is about $6 \%$ lower at $\phi=\pi / 2$ compared with $\phi=0$. Thus, the above discussed power deposition induces a toroidally dependent perpendicular pressure, as the resonant particles are mainly trapped particles with large perpendicular velocities. These trapped particles do not propagate as fast and as freely along the toroidal angle as passing particles do, which is why the parallel pressure does not show any toroidal dependence. Note also that the parallel pressure is about one order of magnitude lower than perpendicular pressure, such that the anisotropy is $T_{\perp} / T_{\|} \approx$ 10 , demonstrating that equilibrium changes are dominated by 

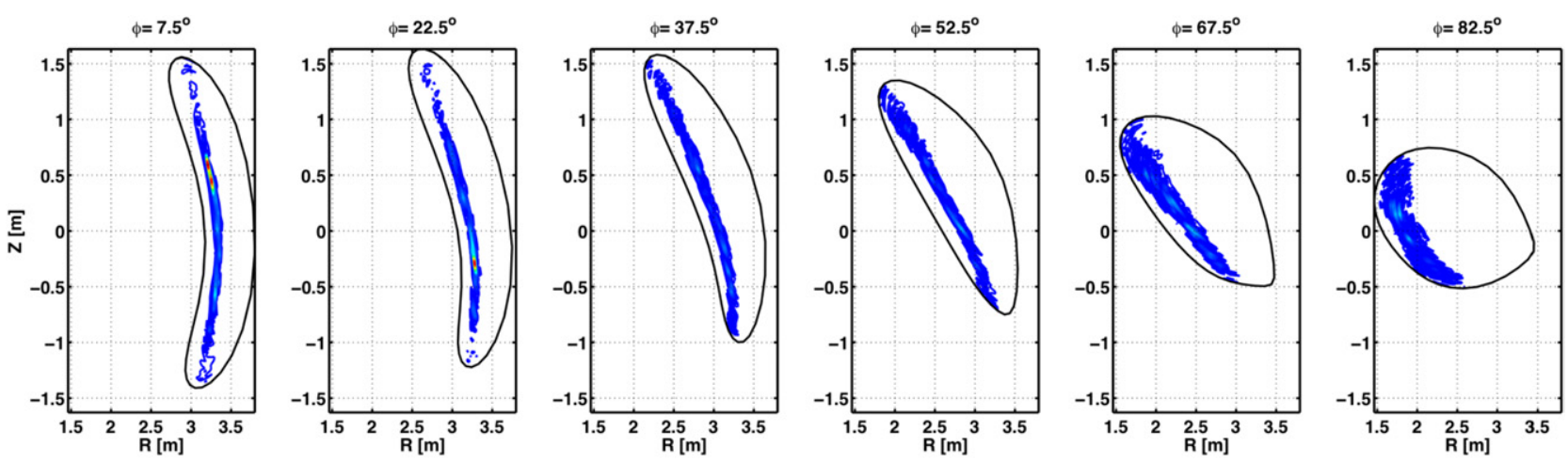

(a) Initial power deposition. An absolute maximum can be found at $\phi \approx 0$, but the local maxima at $\phi \approx \pi / 2$ are easily distinguishable as well.
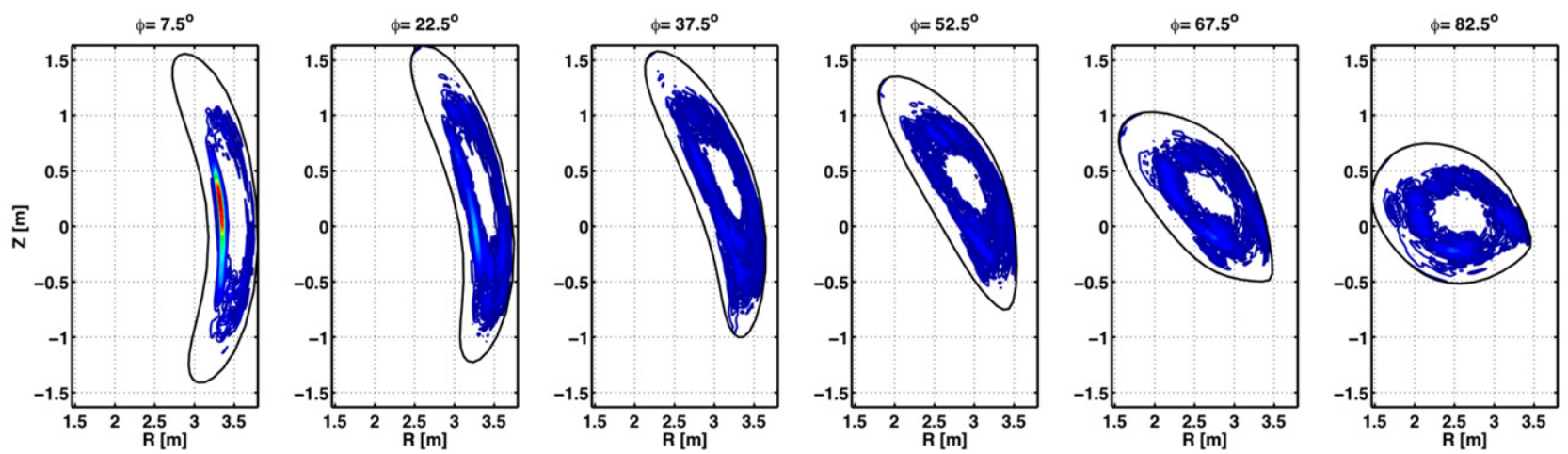

(b) Final power deposition. The absolute maximum around $\phi \approx 0$ is dominant over any other toroidal position. Colors are to scale in all subplots.

Figure 7. Power deposition along the toroidal angle. The toroidal differences increase between the initial and the final state. In the final state, almost all power is absorbed around $\phi=\{0, \pi\}$. The ring shaped absorption comes from fast passing ions with a large Doppler shift $k_{\|} v_{\|}$.

perpendicular heating, and that anisotropic pressure models are imperative to studying RF heating scenarios. The thermal pressure does not show the toroidal dependence of its hot counter part, and therefore the variation in hot perpendicular pressure is decreased when considering total pressure, which is more important in the equilibrium calculations than the hot pressure. Figure 9 compares surface plots of the relative changes of the total perpendicular pressure between $\phi=0$ and $\phi=\pi / 2$. The maximum relative change of total perpendicular pressure during the simulation is an increase of 33\% at $\phi=0$, and $31 \%$ at $\phi=\pi / 2$. Perpendicular pressure is mostly carried by trapped resonant particles, which tend to align their turning points with the resonance [19,22]. Thus, largest pressure variations are in perpendicular direction, and are localized along the resonant layer. We can now generalize these findings from tokamaks to stellarators by stating that the resonant particles not only align their turning points along the resonant layer in the poloidal plane, but also in the toroidal direction. As a result, pressure variations are predominantly in the perpendicular direction, and localized around regions of maximum power deposition in the poloidal plane as well as in toroidal direction. Parallel pressure arises either from passing particles or trapped particles away from the turning points. As resonant particles rarely are passing (except nonstandard orbits at high RF power), the latter effect has to produce most of the hot parallel pressure. As a result, the maximum changes to the parallel pressure of $7 \%$ (not shown) are found on the opposite side of the resonant layer with respect to the magnetic axis, and do not show the toroidal dependence of the perpendicular pressure variations.

This overall increase as well as slight variations along toroidal angle in total pressure caused by a large increase in hot perpendicular pressure must result in a local decrease in magnetic field strength in the MHD equilibrium, and may affect stability. This occurs because of the diamagnetic effect of the pressure on the magnetic field. As the pressure builds up locally, perpendicular pressure gradients become important. These gradients are expected to influence MHD instabilities and particle orbits, e.g. through the toroidal drift frequency $[18,23-25]$.

The perpendicular pressure has a direct effect on the magnetic field strength in the MHD equilibrium, which is illustrated in figure 10. It is still interesting to note that the decrease in magnetic field strength is larger around $\phi=0$ than around $\phi=\pi / 2$, as expected from the corresponding perpendicular pressure variations. However, the magnetic field variations due to RF heating, of the order of $0.2 \%$, are much lower than the pressure variations and cannot be expected to influence particle orbits or wave propagation directly. This is of course explained with the rather low beta in these simulations. We note that for our simulations, variations in magnetic field strength due to hot particle pressure increase are of the same order as variations to the safety factor coming from induced current.

The previously mentioned JET simulations [17] suggest that the effects of heating are locally enhanced by applying the 


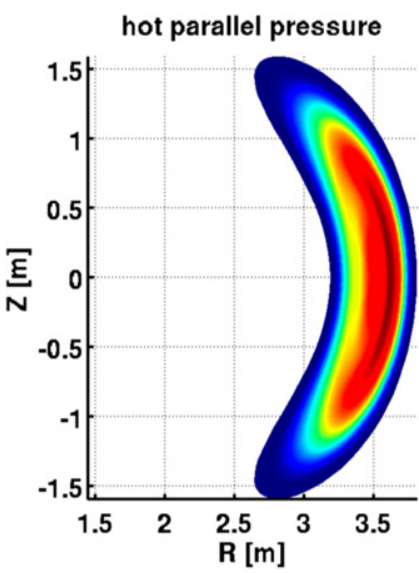

(a) $p_{\|}(\phi=0)$

hot perpendicular pressure

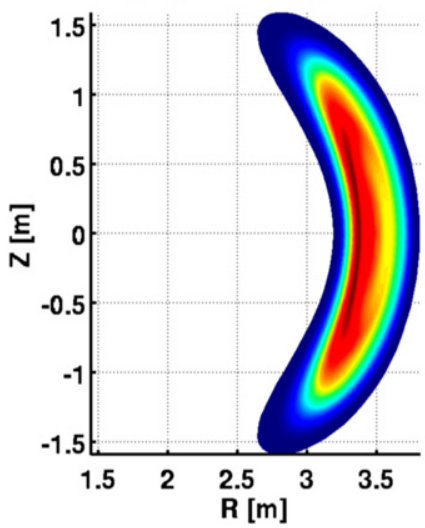

(d) $p_{\perp}(\phi=0)$

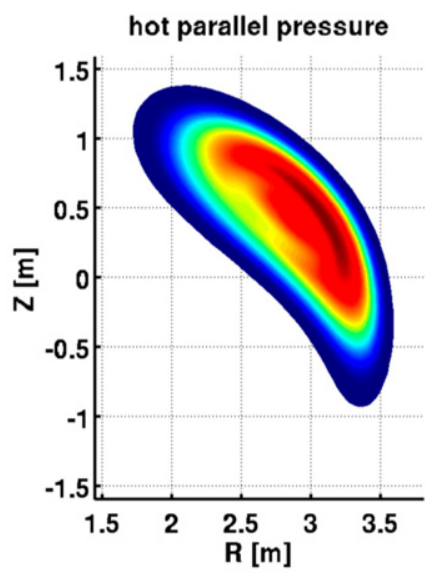

(b) $p_{\|}(\phi=\pi / 4)$

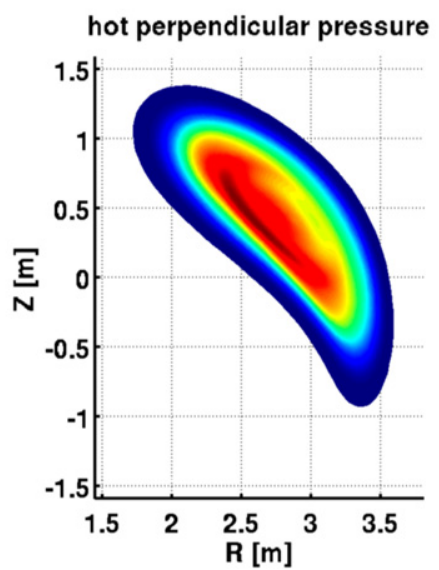

(e) $p_{\perp}(\phi=\pi / 4)$

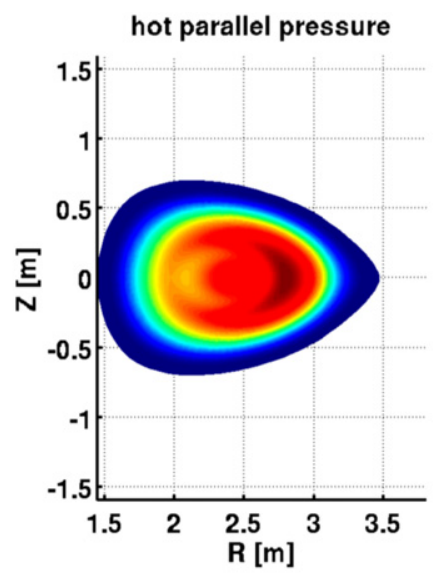

(c) $p_{\|}(\phi=\pi / 2)$

hot perpendicular pressure

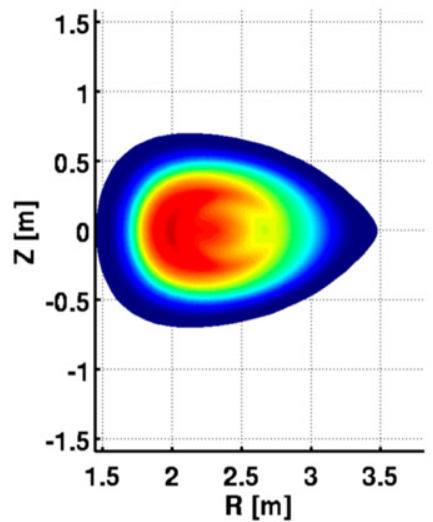

(f) $p_{\perp}(\phi=\pi / 2)$

Figure 8. Toroidal hot parallel and perpendicular pressure. Parallel pressure shows no significant variations along the toroidal angle, whereas the hot perpendicular pressure peak is about $6 \%$ lower at $\phi=\pi / 2$ compared with $\phi=0$. Colours are to scale for parallel and perpendicular pressure separately. Perpendicular pressure is about one order of magnitude larger than parallel pressure, the peak of the former corresponds to $\beta_{\max }^{h} \approx 0.2 \%$.

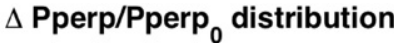

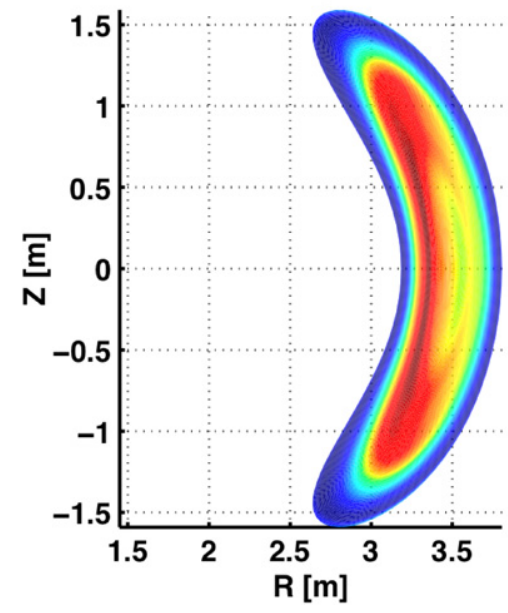

(a) $\Delta p_{\perp}, \phi=0$.

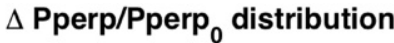

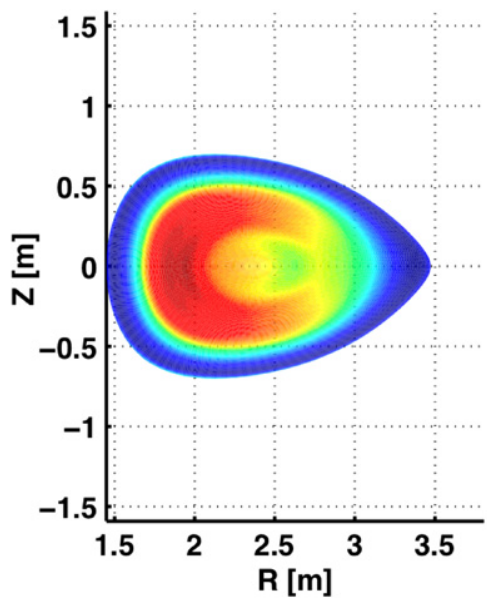

(b) $\Delta p_{\perp}, \phi=\pi / 2$.

Figure 9. Total perpendicular pressure changes at $\phi=\{0, \pi / 2\}$. Plotted is $\left(p_{\perp}-p_{\perp}^{0}\right) / p_{\perp}^{0}[\%]$, where superscript ' 0 ' denotes the initial value (where hot pressure is zero). Colours are to scale, and the peak corresponds to a $33 \%$ change. The peak value decreases to about $31 \%$ between $\phi=0$ and $\phi=\pi / 2$. 


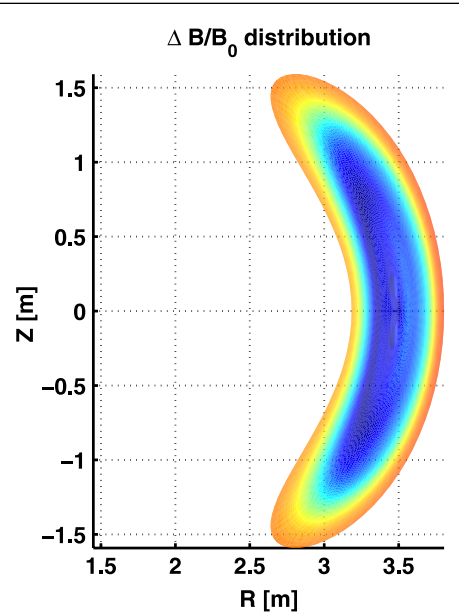

(a) $\phi=0$.

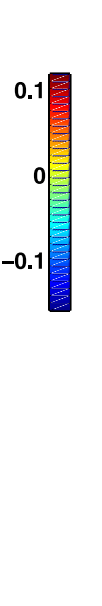

$\left(B-B^{0}\right)$

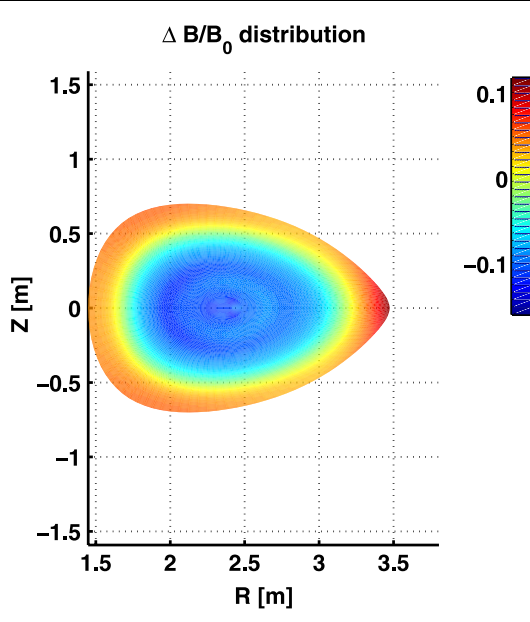

(b) $\phi=\pi / 2$.

Figure 10. Relative modification of the magnetic field strength $\left.\left(B-B^{0}\right) / B^{0}\right)$ in the HFS 6 MW heating scenario. Superscript 0 denotes the initial condition (thermal minority), and the scale is in per cent. Negative values are clearly linked to the positive perpendicular pressure variations of figure 9 , including a small toroidal dependence. Positive values are due to the numerical constraint that total toroidal magnetic flux remains constant during the simulation. Magnetic field strength varies much less than pressure because of the rather low $\beta$ in these simulations.

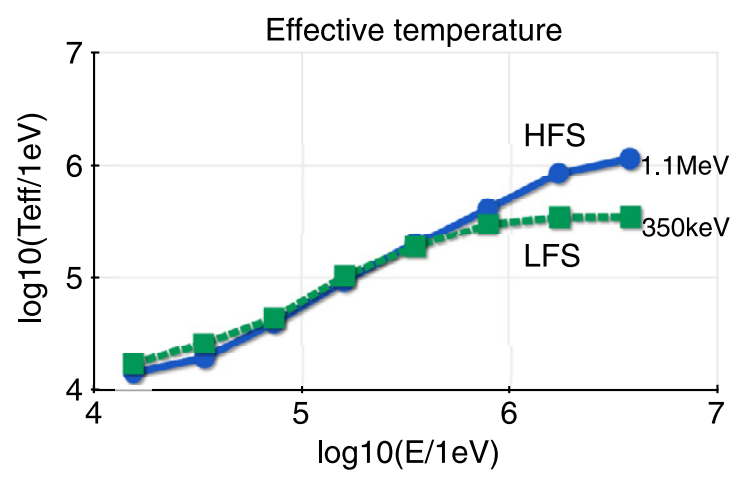

Figure 11. Effective temperatures for the $3 \mathrm{MW}$ HFS and LFS simulations. Whereas the tail reaches $1.1 \mathrm{MeV}$ for HFS, LFS heating creates tails of only about $350 \mathrm{keV}$.

RF power to the LFS instead of the HFS. We will now turn to LFS heating.

\subsection{Low-field side}

Other than in the two-dimensional JET scenarios of [17], LFS heating does not have a greater impact on the magnetic equilibrium than HFS heating in the QAS. Indeed, already at $3 \mathrm{MW}$ heating, most of the resonant particles are very quickly leaving the plasma volume, and the helium-3 minority remains largely thermal. Input power is almost entirely equalized by particle loss even for $3 \mathrm{MW}$, and we will not discuss a $6 \mathrm{MW}$ LFS heating scenario, as the energy content of the minority distribution cannot be expected to increase. Due to high energy particle loss, only about $1 \%$ of the ${ }^{3} \mathrm{He}$ minority gets heated to non-thermal energies and remains confined. Not only is the fraction of highly energetic particles small, but also the tail temperature is considerably lower, as shown in figure 11. When plotting the distribution function contour plots in figure 12, we see that the only high energy particles confined in the plasma are deeply trapped. These particles have smaller orbit widths than barely trapped particles, and are less likely to be lost. In addition, the quasi-axisymmetric concept has a magnetic field structure in Boozer coordinates that makes it look like a tokamak from a guiding centre particle perspective. Small irregularities that cause symmetry breaking could affect mainly the barely trapped particles to develop superbanana orbits, while the deeply trapped fraction would remain unaffected and therefore well confined. This last point only applies to the QAS, and thus results could differ in alternate stellarator systems. Interestingly, even if the percentage of non-thermal minority and the tail temperatures are lower in the LFS case compared with the HFS, the toroidal current density is only about a factor of two smaller than in the HFS scenario (figure 13). As the distribution is dominated by trapped particles, the current density shows the familiar dipolar structure, with a negative current towards the axis, and a positive current further outside [26, 27]. However, the current is now too low to have a visible effect on the safety factor profile, and both the smaller tail temperatures and the small hot particle fraction result in small hot particle pressure, such that the hot particle contribution to the total pressure is negligible. The same applies then to the magnetic equilibrium, where practically no changes are detectable. Instead of considering the relative pressure variations as for the HFS cases above, we plot the locations of high power loss in figure 14, and compare with the magnetic field strength in figure 15. Power loss has a maximum around the toroidal position $\phi=0$ and $\phi=2 \pi / L=\pi$, where the curvature and magnetic field are largest in the plasma. A clear maximum occurs at $\theta \lesssim \pi / 2$, and halfway between the two extremes of the plasma shaping, around $\phi \approx \pi / 4$. When moving in the toroidal direction, any particle feels a minimum magnetic field strength around $\phi=N 2 \pi / 2 L=\{\pi / 2,3 \pi / 2\}$, and a maximum around $\phi=N 2 \pi / L=\{0, \pi\}$, and can be trapped toroidally, with turning points around $\phi=\{0, \pi\}$. Particle drift across flux surfaces is largest around the turning points, which explains why particles are lost mainly around $\phi=\{0, \pi\}$. Now, the trapped particles move parallel to the toroidal magnetic field on the outer leg of their orbits, and in counter direction on 


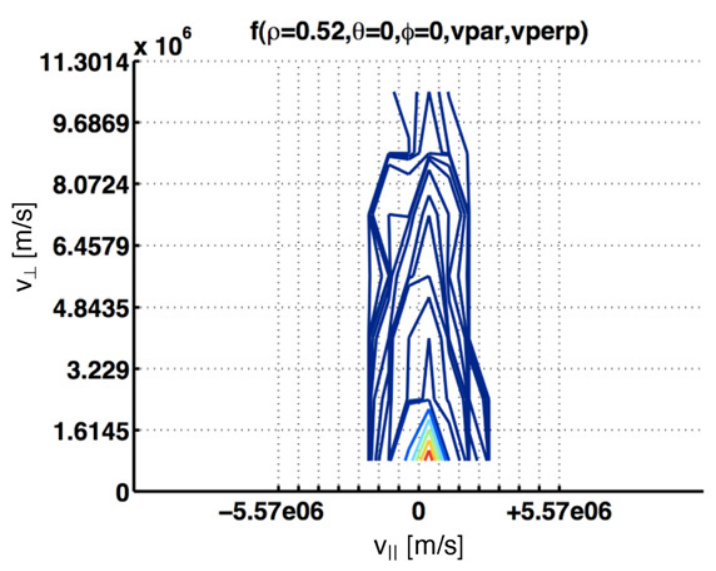

(a) $f\left(v_{\|}, v_{\perp}\right)$ at $\phi=0$.

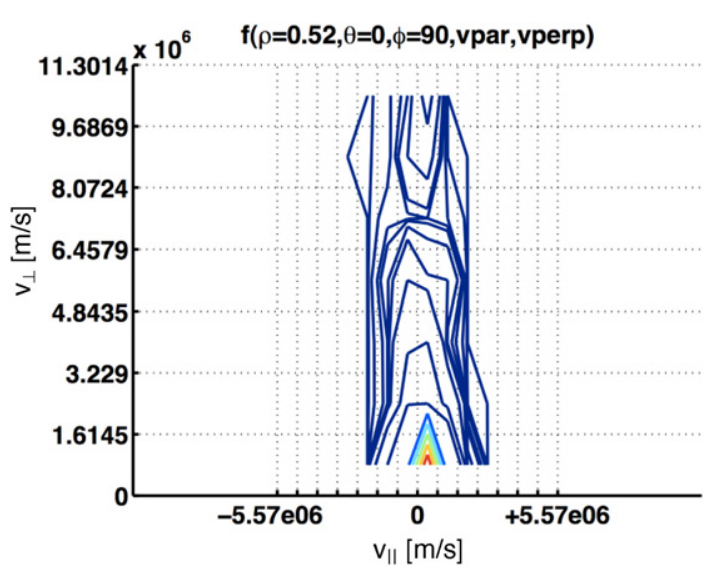

(b) $f\left(v_{\|}, v_{\perp}\right)$ at $\phi=\pi / 2$.

Figure 12. Hot particle distribution function contour plots for 3 MW LFS heating. The distribution function is dominated by deeply trapped particles $\left(\left|v_{\|}\right| \ll v_{\perp}\right)$. These particles have smaller orbit width than barely trapped particles, and stay confined in the plasma, whereas more barely trapped particles are very quickly crossing the plasma boundary.

the inner leg [27]. At the same time, particles are much more likely to hit the plasma edge on the outer leg of their orbit than on the inner leg. As a toroidally trapped particle moves on the inner leg of the orbit, it moves in the negative $\phi$ direction, i.e. from $0(\pi)$ towards $-\pi(0)$. Once $-\pi / 2(\pi / 2)$ passed, the magnetic field strength increases and the particle is more and more likely to reach its turning point. As the magnetic field is strongest around $-\pi(0)$, this will happen before, and the particle will move onto the outer leg of its orbit, where it will either continue along its path and complete the orbit, or be lost before reaching $-\pi / 2(\pi / 2)$, such that on average, most particles will be lost around $-3 \pi / 4=5 \pi / 4(\pi / 4)$. Of course, this description applies only to particles with finite orbit width, and thus not to deeply trapped particles. The latter remain confined, as discussed above (figure 12). We note also that in the QAS we have explored, the symmetry of the magnetic field spectrum in the Boozer coordinate frame is not perfect. This may amplify the effect of the smaller volume (compared with the reference JET configuration) as a cause of the poor confinement properties observed with LFS heating in our simulations and partially explain the difference with previous axisymmetric JET tokamak computations [17].

In order to push magnetic field strength variations, and therefore effects on the overall magnetic equilibrium, different alternatives can be explored. We have tested increasing the HFS RF power even further to $12 \mathrm{MW}$ (with the same minority concentration), but found that with such high power, next to all the resonant particles are crossing the plasma boundary, as particle orbits become very large, and toroidal trapping helps fast particles to escape. If we try to increase hot beta by increasing the initial minority temperature (we tried ten and one hundred times), particles with large perpendicular velocities (i.e. trapped particles) are immediately leaving the plasma volume, thus diminishing hot particle beta and pressure anisotropy, until the hot particles are slowed down enough to represent the same solution we showed above. This behaviour confirms the robustness of our numerical model, as it converges to similar results with different initial conditions. It also strengthens the results of these simulations, that due to toroidal magnetic field variations, much more resonant particles are

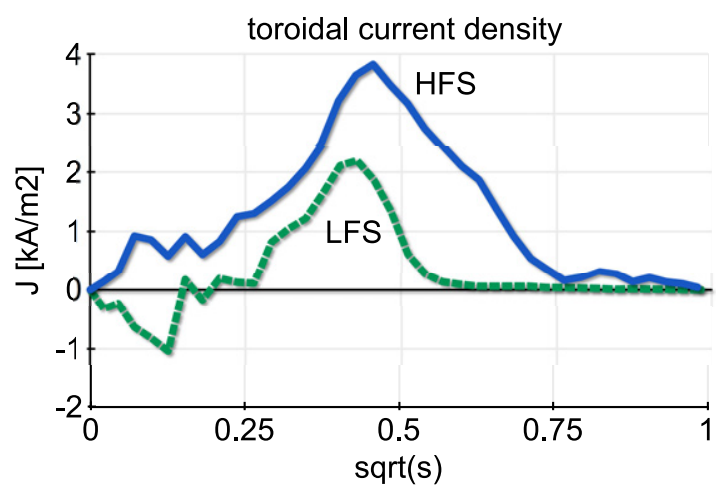

Figure 13. Even if the fraction of hot particles is rather small, the toroidal current density is only about a factor of two smaller in the LFS compared with the HFS case. As by far the largest part of the hot particles are trapped, the current density shows now the characteristic dipolar structure. Other than for the HFS (figure 5), the current density has no visible effect on the safety factor profile.

lost than in comparable tokamaks. Our simulations suggest that ICRH in a JET-like plasma is more efficient than in the here studied QAS configuration.

\section{Conclusions}

For the first time, the code package SCENIC has been applied to a three-dimensional geometry. Studying a twoperiod quasi-axisymmetric stellarator, we could demonstrate that self-consistent solutions for ICRH in three-dimensional plasmas can be obtained, including a variable equilibrium. For high-field side (HFS) high and moderate power simulations using 1\% helium-3 in deuterium, we found that a high energy non-Maxwellian tail forms in the distribution function, accounting for $16 \%(12 \%)$ of the ${ }^{3} \mathrm{He}$ minority for $6 \mathrm{MW}$ (3 MW) RF power, with tail temperatures reaching $1.4 \mathrm{MeV}$ $(1.1 \mathrm{MeV})$. The toroidal current is entirely RF induced, and reaches $4.5 \mathrm{kA}(1 \mathrm{kA})$, resulting in a $0.5 \%(0.1 \%)$ variation of the safety factor profile, because we have neglected the bootstrap current. Resonant particles are trapped due to preferentially perpendicular heating, thus creating important 


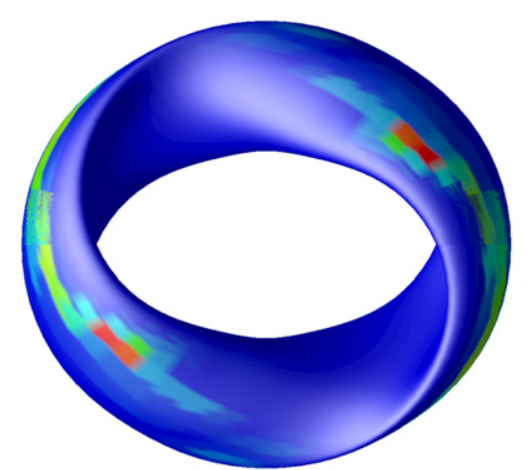

(a) Top view.

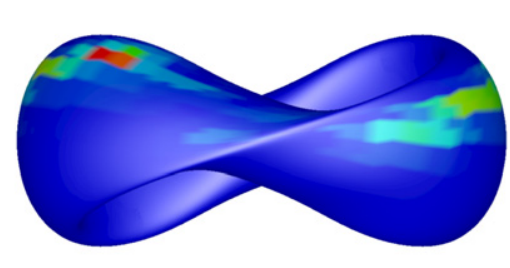

(b) Side view

Figure 14. Three-dimensional plots of the lost power due to particles hitting the plasma edge. Highest loss occurs on the upper LFS of the plasma $(0 \leqslant \theta<\pi / 2)$, and around $\phi=0,2 \pi / L$, where curvature and magnetic field strength is most important. Additional losses occur around $\theta=0$ and $\pi / 2<\phi<\pi$. Both of these locations coincide with increasing magnetic field strength for toroidally trapped particles (figure 15).

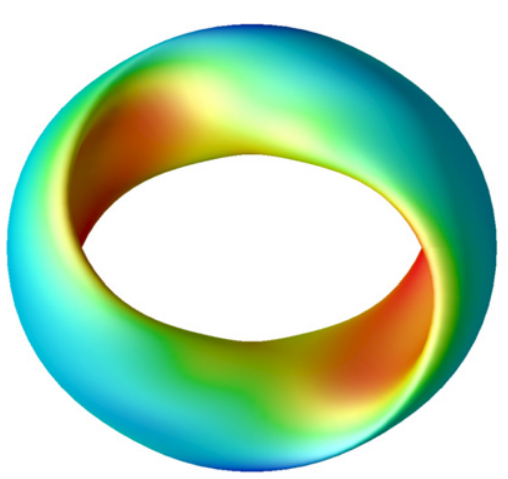

(a) Top view.

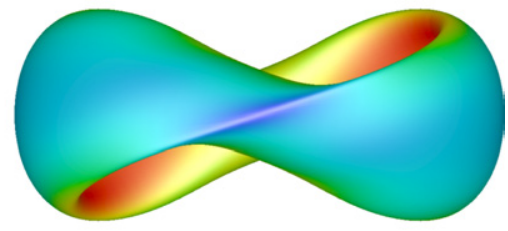

(b) Side view.

Figure 15. Magnetic field strength along the plasma edge. As in tokamaks, for any given toroidal angle, the minimum is found around the midplane $\theta=0$. In toroidal direction, $B$ has a minimum around $\phi=\pi / 2$, such that toroidally trapped particles are most likely to have their turning points (and therefore largest drift across flux surfaces) around $\phi=N 2 \pi / L=\{0, \pi\}$.

pressure anisotropy $T_{\perp} \sim 10 T_{\|}$. As opposed to a tokamak, particles are trapped poloidally and toroidally, introducing a toroidal dependence of power deposition and perpendicular pressure. As ICRH is applied, toroidal power deposition variations cause more resonant particles to become toroidally trapped, in turn causing larger toroidal power deposition variations on the one hand and enhanced power loss due to highly energetic particle leaving the plasma volume on the other hand. In the HFS heating scenarios, 60\% (50\%) of the input power is lost for $6 \mathrm{MW}(3 \mathrm{MW})$, decreasing $\mathrm{RF}$ heating efficiency considerably. Growing hot (perpendicular) pressure is responsible for increasing total pressure by as much as $30 \%(6 \mathrm{MW})$, and creates a magnetic well around the resonant layer. This change is of the order of $0.1 \%$ in these low beta simulations, and is of the same order as safety factor profile modifications due to induced toroidal current. Higher RF power or higher initial minority temperature leads to heavily increased particle loss, which is also observed in 3 and $6 \mathrm{MW}$ low-field side (LFS) heating simulations. Here, nearly all power is lost already at $3 \mathrm{MW}$, indicating that such heating scenarios are very inefficient. In contrast to the JET studies of [17], important fast toroidally trapped particle loss generates lower tail temperature, current and pressure for LFS compared with HFS heating, suggesting that lowfield side heating with low minority concentration may not be appropriate for efficiently heating plasma configurations similar to the here considered quasi-axisymmetric stellarator. Confinement properties can be expected to improve markedly by increasing the helium- 3 concentration (but remaining in a minority scheme) on the one hand, and the plasma volume on the other hand. In such geometries, effects on the magnetic equilibrium could be more important than in this work. It might also be worthwhile including modifications of the thermal ion and electron profiles, e.g. for adding bootstrap current modifications in the self-consistent simulations. These possibilities will be explored in the future.

\section{Acknowledgments}

All presented simulations have been performed on the Rosa XT5 supercomputer at the Swiss National Supercomputing Centre (CSCS) in Manno, Switzerland. This work was supported in part by the Swiss National Science Foundation.

\section{References}

[1] Boozer A.H. 1998 Phys. Plasmas 51647

[2] Boozer A.H. 2008 Plasma Phys. Control. Fusion 50124005

[3] Hedin J., Hellsten T., Eriksson L.-G. and Johnson T. 2002 Nucl. Fusion 42527 
[4] Brambilla M. and Bilbato R. 2009 Nucl. Fusion 4985004

[5] Jaeger E.F. et al 2006 Nucl. Fusion 46 S397

[6] Choi M. et al 2010 Phys. Plasmas 1756102

[7] Murakami S. et al 2006 Nucl. Fusion 46 S425

[8] Jucker M. et al 2011 Comput. Phys. Commun. 182912

[9] Hirshman S.P. and Betancourt O. 1991 J. Comput. Phys. 9699

[10] Cooper W.A. et al 2009 Comput. Phys. Commun. 1801524

[11] Popovich P., Cooper W.A. and Villard L. 2006 Comput. Phys. Commun. 175250

[12] Mellet N. et al 2011 Comput. Phys. Commun. 182570

[13] Fischer O., Cooper W.A., Isaev M.Y. and Villard L. 2002 Nucl. Fusion 42817

[14] Cooper G.A. et al 2007 Phys. Plasmas 14102506

[15] Okamura S. et al 1998 J. Plasma Fusion Res. 1164

[16] Cooper W.A. et al 2006 Nucl. Fusion 46683
[17] Jucker M., Graves J.P., Cooper W.A. and Johnson T. 2011 Plasma Phys. Control. Fusion 53054010

[18] Jucker M., Graves J.P., Cooper G.A. and Cooper W.A. 2008 Plasma Phys. Control. Fusion 5065009

[19] Hellsten T. et al 2004 Nucl. Fusion 44892

[20] Madden N.A. and Hastie R.J. 1994 Nucl. Fusion 34519

[21] Cooper W.A. et al 2007 Plasma Phys. Control. Fusion 491177

[22] Eriksson L.-G. et al 1998 Phys. Rev. Lett. 811231

[23] Connor J.W., Hastie R.J. and Taylor J.B. 1978 Phys. Rev. Lett. 40396

[24] McClements K.G., Dendy R.O., Hastie R.J. and Martin T.J. 1996 Phys. Plasmas 32994

[25] Bourdelle C. et al 2005 Nucl. Fusion 45110

[26] Hellsten T., Carlsson J. and Eriksson L.-G. 1995 Phys. Rev. Lett. 743612

[27] Carlsson J., Hellsten T. and Hedin J. 1998 Phys. Plasmas 52885 